\title{
Differentiation of immature DCs into endothelial-like cells in human esophageal carcinoma tissue homogenates
}

\author{
JING LU $^{1 *}$, RUIHUA BAI $^{2 *}$, ZHENZHU QIN $^{3 *}$, YANYAN ZHANG $^{1}$, XIAOYAN ZHANG ${ }^{1}$, \\ YANAN JIANG ${ }^{1}$, HONGYAN YANG ${ }^{1}$, YOUTIAN HUANG ${ }^{1}$, GANG LI $^{4}$, MINGYAO ZHAO ${ }^{1}$ and ZIMING DONG ${ }^{1}$ \\ ${ }^{1}$ Department of Pathophysiology, School of Basic Medical Sciences, Zhengzhou University, Zhengzhou 450001; \\ ${ }^{2}$ Department of Pathology, Henan Tumor Hospital, Zhengzhou 450003; ${ }^{3}$ Department of Pathology, The First People's \\ Hospital of Shangqiu, Shangqiu 476100, P.R. China; ${ }^{4}$ Department of Dermatology and Skin Science, Research \\ Pavilion, Vancouver Coastal Health Research Institute, University of British Columbia, Vancouver, BC, Canada
}

Received April 3, 2013; Accepted May 2, 2013

DOI: 10.3892/or.2013.2491

\begin{abstract}
We previously reported endothelial-like differentiation (ELD) of immature dendritic cells (iDCs) in the microenvironment derived from EC9706 human esophageal squamous cell carcinoma conditioned medium (CM). However, the $\mathrm{CM}$ is far different from the esophageal carcinoma tissue of patients. In addition, the potential role of peri-esophageal carcinoma in the ELD of iDCs is also unknown. In the present study, we showed that the tumor microenvironment derived from esophageal carcinoma homogenate promoted iDCs to differentiate from the DC pathway toward endothelial cells, while the peri-esophageal carcinoma homogenate did not have this function. During the course of ELD, ERK signaling pathway and CREB were activated. Blocking MEK, both the phosphorylation of ERK and CREB, and the ELD of iDCs were inhibited. These data suggest that esophageal carcinoma tissue, not peri-esophageal carcinoma tissue, can drive iDCs to differentiate into endothelial-like cells, instead of differentiation into mature DCs, thereby losing the ability of antigen presentation.
\end{abstract}

\section{Introduction}

Dendritic cells (DCs) are antigen-presenting cells (APCs) that show the unique ability to activate and regulate immune responses (1). DCs can provide antigenic peptides to initiate naive T-cell responses, as well as produce potent costimulatory molecules to promote T-cells differentiation $(2,3)$. Plenty

Correspondence to: Professor Ziming Dong, Department of Pathophysiology, School of Basic Medical Sciences, Zhengzhou University, Zhengzhou 450001, P.R. China

E-mail: dongzm@zzu.edu.cn

${ }^{*}$ Contributed equally

Key words: esophageal carcinoma, tissue homogenate, dendritic cells, endothelial-like differentiation of studies have demonstrated that both the cell surface markers and antigen presenting function of tumor-infiltrating DCs decreased significantly $(4,5)$. However, the mechanisms during this process are still to be illustrated.

Some research has shown that DC precursors can transdifferentiate into endothelial-like cells (ELCs) in mouse and human ovarian carcinomas indicating a role of VEGF-A and $\beta$-defensins (6). CD34+ progenitor cells redirected their differentiation into endothelial cells (ECs) from developing into DCs under the influence of conditioned medium (CM) of murine Lewis lung carcinoma cells (7). In addition, tumorassociated DCs incubated with the pro-angiogenic factors VEGF and oncostatin M, trans-differentiated into ELCs, suggesting an alternative pathway of tumor angiogenesis (8). The research shows that DC progenitor or immature DCs (iDCs) can trans-differentiate into ELCs, possibly contributing to vasculogenesis. Thereby, DCs might exert some impact on the neovascularization process in different physiopathological conditions (9).

Esophageal cancer is one of the most frequently diagnosed cancers in Asia and has high-incidence and high mortality rate. Esophageal squamous cell carcinoma is the main type of esophageal cancer, which has strong invasiveness and poor prognosis. We previously reported that the CM from human esophageal squamous cell carcinoma (ESCC) cell line EC9706 induced iDCs to differentiate into ELCs (10). However, this CM is far different from the esophageal carcinoma tissue of patients. The potential role of peri-esophageal carcinoma in the endothelial-like differentiation (ELD) of iDCs is also unknown. In the present study, we examined the possibility of ELD of iDCs in the microenvironment derived from the tissue homogenate of esophageal carcinoma and peri-esophageal carcinoma, and investigated the role of extracellular signal-regulated kinase (ERK) signaling and cAMP response element binding protein $(\mathrm{CREB})$ in this process.

\section{Materials and methods}

Preparation of tissue homogenate supernatant of human esophageal carcinoma and peri-carcinoma. Fresh tumor 
specimens of ESCC tissue and peri-carcinoma tissue $(>5 \mathrm{~cm})$ 6 cases (without preoperative chemotherapy and radiotherapy) were collected to prepare to the tissue homogenate supernatant by grinding and centrifuge. The above specimens were contributed by donors from the Henan Tumor Hospital.

Cell culture. The culture of primary human umbilical vein endothelial cells (HUVECs) is according to our previous report (10). The aseptic cords were contributed by healthy parturient donors from the Third Affiliated Hospital, Zhengzhou University. Peripheral blood mononuclear cells (PBMCs) were harvested from healthy adult volunteers by density gradient centrifugation over Ficoll (8) and seeded in 24-well plates at $2 \times 10^{9} / 1$ for $3 \mathrm{~h}$. The adherent cells (monocytes) were induced toward DCs with rhGM-CSF $100 \mu \mathrm{g} / 1$ (Amoytop), rhIL-4 $5 \mu \mathrm{g} / \mathrm{l}$ (PeproTech). ESCC homogenate supernatant (40\%) or peri-carcinoma homogenate supernatant $(40 \%)$ was added at the end of day 2. After 7 days induction, induced cells were harvested on day 9 (i.e. 2+7) for study. Parallel culturing of control DCs also were harvested on day 9.

Western blot analysis. Total protein concentration of each group of cells was measured by the Bradford method. Cell lysates $(50 \mu \mathrm{g})$ were resolved to $10 \%$ SDS-PAGE gel and transferred to PVDF membrane. CD144 Ab (1:1000; Cell Signaling Technology), vWF Ab (1:300), phospho-p44/42 MAPK/ERK Ab (Thr202/Tyr204; 1:1000; Cell Signaling Technology), p44/42 MAPK/ERK Ab (Thr202/Tyr204; 1:1000; Cell Signaling Technology) were added, respectively, overnight at $4^{\circ} \mathrm{C}$. HRP-IgG secondary antibody was incubated for $2 \mathrm{~h}$ at room temperature. The membranes were visualized with ECL. The Gel Doc Imaging system was used for detecting the gray value of the protein bands.

Immunofluorescence. Control DCs, induced cells, and the positive control HUVECs were seeded in 96-well plate and incubated for $24 \mathrm{~h}$, then fixed with $4 \%$ paraformaldehyde for $30 \mathrm{~min}$. Rabbit anti-human vWF antibody (1:200; Santa Cruz Biotechnology) and FITC-conjugated goat anti-rabbit IgG antibody were used. The procedure was followed by an immunocytochemical protocol. Cells with green fluorescent particles in the cytoplasm were counted as having positive expression. The Biosens Digital Imaging system was used for detecting the fluorescence intensity of the samples.

Dil-Ac-LDL and India ink uptake assay $(8,11)$. The induced cells and control groups were seeded in 96-well plate and incubated for $36 \mathrm{~h}$. Dil-Ac-LDL $(10 \mu \mathrm{g} / \mathrm{ml})$ (Biomedical Technologies Inc.) was added and incubated at $37^{\circ} \mathrm{C}$ for $4 \mathrm{~h}$. The medium containing Dil-Ac-LDL was removed, and washed 3 times with PBS. India ink $(10 \mu \mathrm{l} / \mathrm{ml})$ was added and the cells were incubated at $37^{\circ} \mathrm{C}$ for another $4 \mathrm{~h}$. The cells were washed 3 times with PBS and observed with a fluorescence microscope.

Preparation of whole-cell antigen.EC9706 cells were collected and washed twice with PBS. Resuspended to a concentration of $1 \times 10^{10} / 1$. The antigen was prepared by ultrasonic crushing technique for $3 \mathrm{~min}$ (pulse $4 \mathrm{sec}$, amplitude 1) and centrifuged $13,000 \mathrm{rpm} / \mathrm{min}$ for $30 \mathrm{~min}$ at $4^{\circ} \mathrm{C}$. The supernatant was collected and filtered. Antigen concentration was determined by Bradford method and stored at $-20^{\circ} \mathrm{C}$ for use.

Mixed lymphocyte reaction. EC9706 cell antigen was added into the cells on day 4 at the final concentration $(100 \mathrm{mg} / \mathrm{l})$ and these cells were collected on day 9. Pulsed cells were regarded as stimulator cells, and the autologous T cells were regarded as response cells, and they were mixed in 1:20 ratios. The mixed cells were placed in 96 -well plate $\left(1 \times 10^{4} /\right.$ well) in quadruplicate for $72 \mathrm{~h}$. CCK-8 (10 $\mu \mathrm{l} /$ well) (Dojindo Laboratories) was incubated with the mixed cells for $2 \mathrm{~h}$ and the absorbance was measured at $450 \mathrm{~nm}$. The proliferation rate of the T cells was defined as $(\%)=($ A value of experimental group - A value of quiescent $\mathrm{T}$ cells)/(A value of quiescent $\mathrm{T}$ cells) $\mathrm{x} 100 \%$.

Cytotoxicity assay. Autologous T cells and pulsed cells were co-cultured in a ratio of 20:1 for $72 \mathrm{~h}$. CTLs and EC9706 cells were placed in 96-well plate in quadruplicate for $72 \mathrm{~h}$, according to the ratio of $30: 1$, in a total volume of $200 \mu \mathrm{l} /$ well (1x10 $/$ well). CTL killing activity was detected by CCK-8 kit (Dojindo Laboratories) according to the protocol. The CTL killing ratio was defined as $(\%)=(\mathrm{A}$ value of the control group - A value of the experimental group)/(A value of the control group) $\mathrm{x} 100 \%$.

Statistical analysis. Data were expressed as means \pm standard deviation (SD) with at least three separate experiments and analyzed by one-way ANOVA and q-test. Significance was defined as $\mathrm{P}<0.05$.

\section{Results}

The changes of morphology and cell marker of the ELD of iDCs in tissue homogenate supernatant of esophageal carcinoma and peri-carcinoma. The iDCs were induced by esophageal carcinoma or peri-carcinoma homogenate for 7 days. The cells induced by carcinoma homogenate were slender, while most of the cells induced by peri-carcinoma homogenate were round, similar to the appearance of control DCs (Fig. 1).

The expression levels of endothelial cell markers vWF and CD144 were significantly increased in cells induced by carcinoma homogenate compared with the peri-carcinoma group $(n=3, P<0.001, P<0.01$, respectively) and control DCs $(n=3$, $\mathrm{P}<0.001, \mathrm{P}<0.01$, respectively). There was no obvious difference between the peri-carcinoma group and control DCs $(n=3$, $\mathrm{P}>0.05$ ) (Fig. 2A). Immunofluorescence analysis also revealed the enhanced expression of vWF in carcinoma homogenate induced cells compared with the peri-carcinoma group $(n=3$, $\mathrm{P}<0.01)$ and control DCs $(\mathrm{n}=3, \mathrm{P}<0.01)$ (Fig. 2B).

The functional changes of the ELD of iDCs in tissue homogenate supernatant of esophageal carcinoma and pericarcinoma. Uptake of Dil-Ac-LDL is considered to be one of the typical functions of ECs although shared with other cells as macrophages and monocytes $(12,13)$. However, monocytes and macrophages show uptake of Dil-Ac-LDL as well as India ink (11). In previous research we have proved that PBMCs had weak uptake of Dil-Ac-LDL, and strong uptake of India ink (10). In the present study, the induced cells by esophageal carcinoma homogenate did not intake India ink 

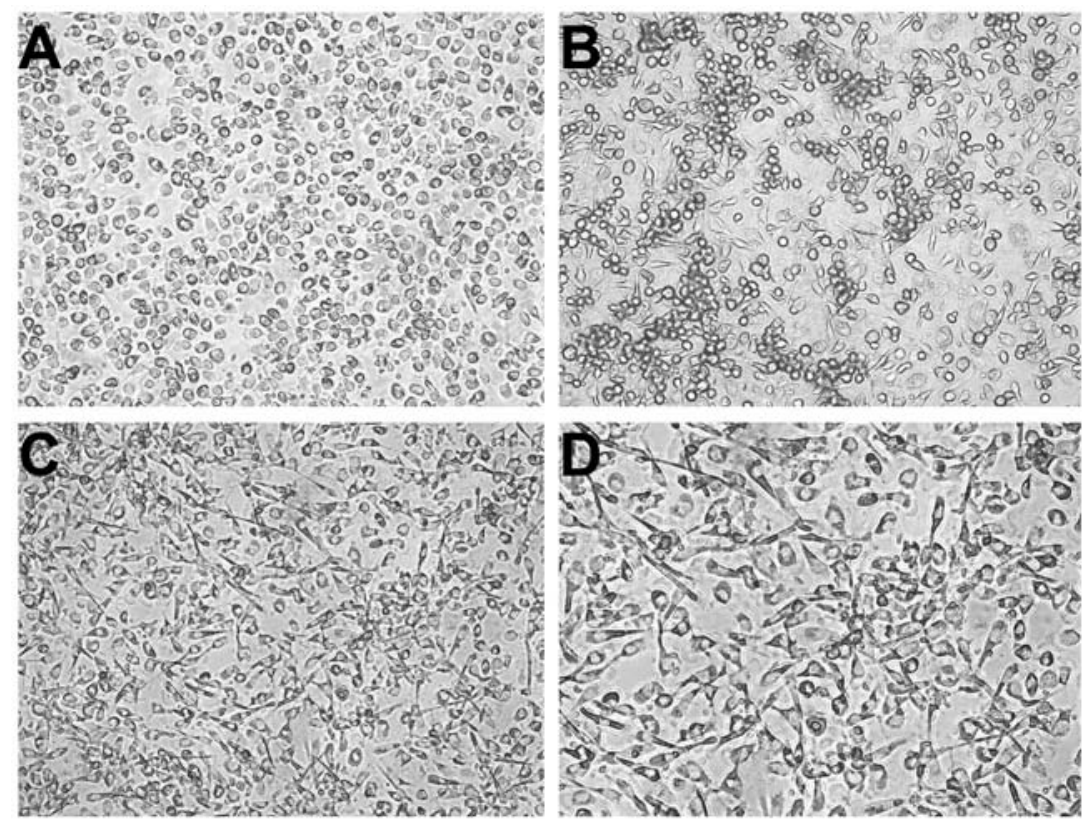

Figure 1. Cell morphology of the ELD of iDCs induced by esophageal carcinoma tissue homogenate supernatant. PBMCs were incubated in rhGM-CSF/rhIL-4, and the $40 \%$ homogenate supernatant of esophageal carcinoma or peri-carcinoma was added at the end of day 2 . The cells of esophageal carcinoma group appeared with fusiform shape after 7-day induction (C and D). The cells of the peri-carcinoma group (B) had no obvious morphology change, similar to control DCs (A). (Original magnification, $\mathrm{x} 200$ for A-C, and $\mathrm{x} 400$ for D).
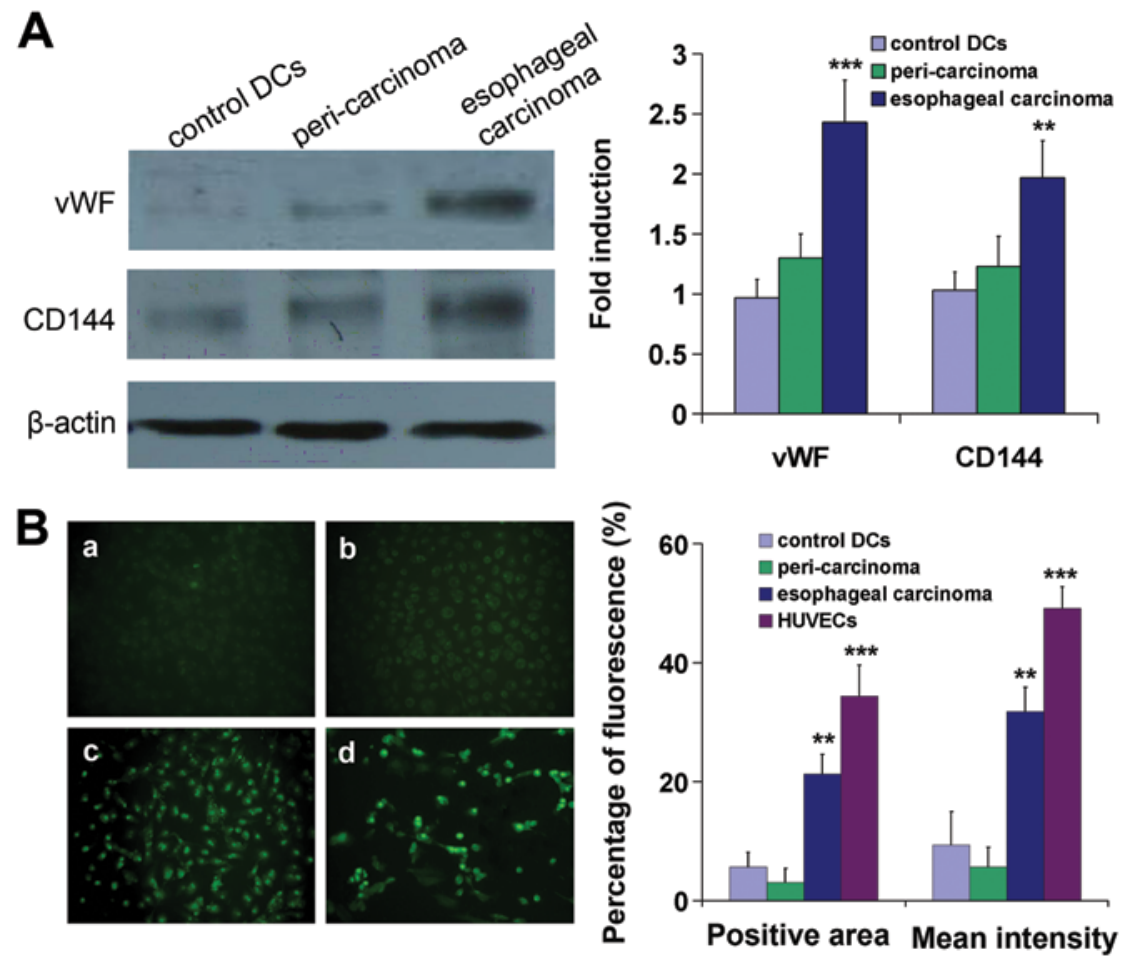

Figure 2. EC markers vWF and CD144 increased after induction by esophageal carcinoma tissue homogenate supernatant. (A) Western blot detection the protein levels of vWF and CD144 in the induced cells. (B) Immunofluorescence detection of the expression of vWF in control DCs (a), peri-carcinoma group cells (b), esophageal carcinoma group cells (c), and positive control HUVECs (d). (Original magnification, $x 200$ ). Results shown are mean $\pm \mathrm{SD}(\mathrm{n}=3)$. ${ }^{* *} \mathrm{P}<0.01$, ${ }^{* * *} \mathrm{P}<0.001$ vs. control DCs and peri-carcinoma.

(Fig. 3 right panel), excluding the possibility that macrophages and monocytes were mixed in with the induced cells. The obviously increased uptake of Dil-Ac-LDL in induced cells by carcinoma homogenate showed that the iDCs tended to differentiate toward ECs (Fig. 3C). In contrast, there was no difference of Dil-Ac-LDL uptake between peri-carcinoma and control DCs (Fig. 3A and B).

To determine the change of antigen presentation function of iDCs induced by esophageal carcinoma homogenate, we measured their ability to stimulate $\mathrm{T}$ cell proliferation and 

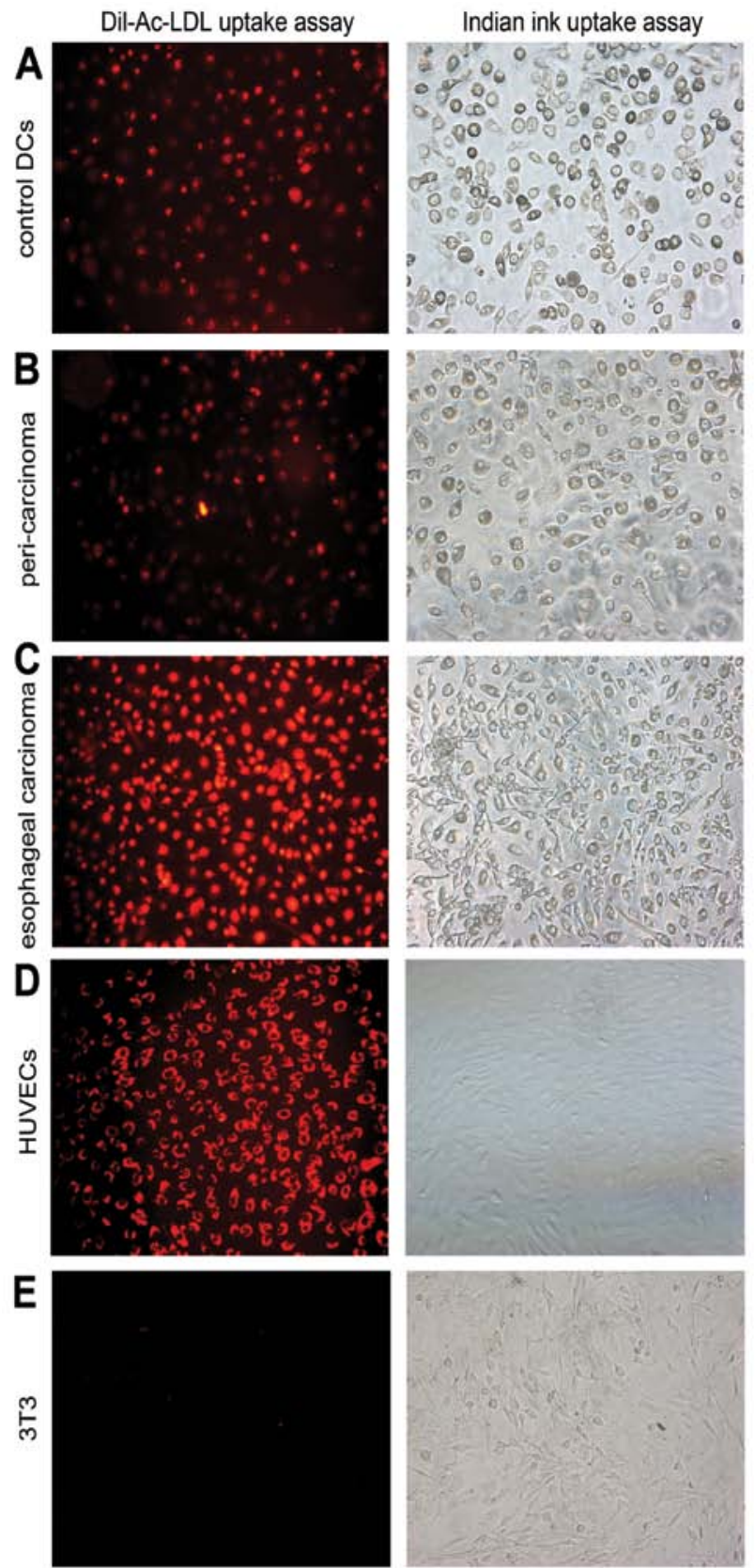

Figure 3. Dil-Ac-LDL and India ink uptake assay. Left panel shows DilAc-LDL uptake, right panel shows no india ink uptake respectively. (Original magnification, $\mathrm{x} 200)$.

CTL activity for killing EC9706 cells in vitro. The results showed that the microenviroment produced by esophageal carcinoma homogenate significantly disabled the antigen presenting function of the cells $(\mathrm{P}<0.01)$, however, the pericarcinoma homogenate did not have this effect on the cells (Fig. 4).

Thus, the above results showed that esophageal carcinoma homogenate, not peri-esophageal carcinoma homogenate, induced iDCs to differentiate into endothelial-like cells, instead of differentiation into mature DCs.

ERK signaling and CREB is involved in ELD of iDCs induced by the esophageal carcinoma tissue homogenate supernatant. In previous research, we found that EC9706 CM
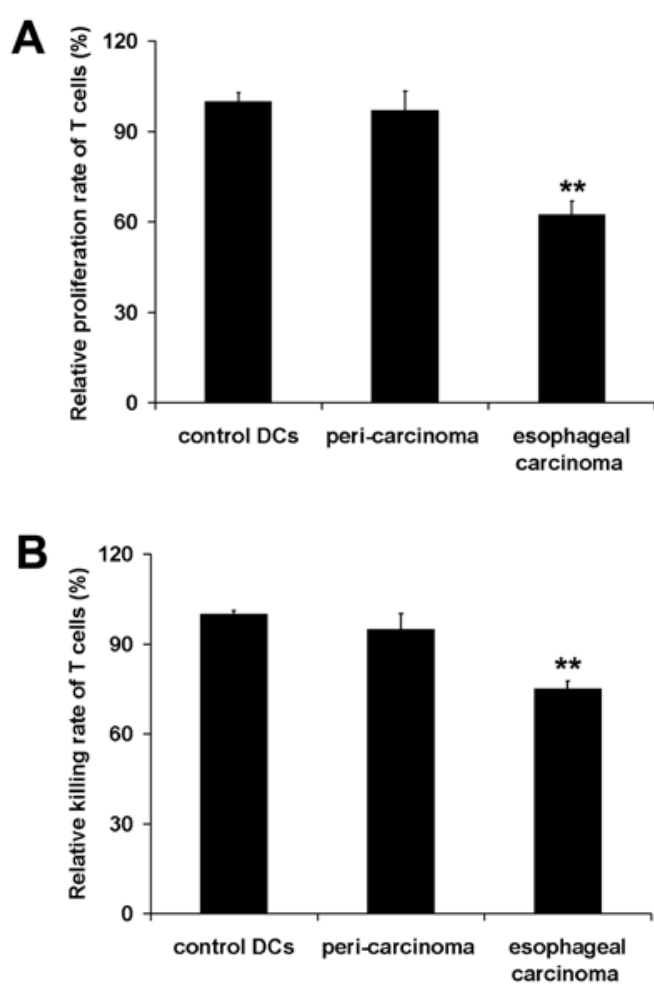

Figure 4. The proliferation rate and killing rate of $\mathrm{T}$ cells $(\%)(\mathrm{n}=4$ means \pm SD). (A) The proliferation rate of the T cells. (B) The killing rate of the $\mathrm{T}$ cells. ${ }^{* *} \mathrm{P}<0.01$ vs. control DCs and peri-carcinoma

activated ERK signaling pathway and CREB to mediate ELD of iDCs (10). Thus, we asked if ERK signaling and CREB are also involved in ELD of iDCs induced by esophageal carcinoma homogenate. We found that the phosphorylation levels of ERK and CREB increased in a time-dependent manner in the differentiating iDCs. Esophageal carcinoma homogenate stimulated strong phosphorylation of ERK after $30 \mathrm{~min}$ of incubation and CREB after $15 \mathrm{~min}$ of incubation. PD98059 is a selective inhibitor of MEK, the upstream regulator of phosphorylation of ERK. Therefore, we used PD98059 $50 \mu \mathrm{M}$ to block MEK to investigate the phosphorylation levels of ERK and CREB. Results showed that the phosphorylation levels of both ERK and CREB were significantly inhibited in the presence of $\mathrm{PD} 98059$ ( $\mathrm{n}=3, \mathrm{P}<0.001$ and $\mathrm{P}<0.01$, respectively) (Fig. 5).

Blocking ERK signaling inhibits ELD of iDCs in the esophageal carcinoma tissue homogenate. After 7 days induction by esophageal carcinoma homogenate, the expression levels of phospho-ERK and CREB were still strong in the differentiating iDCs. In order to clarify that the ERK signaling pathway is essential for the differentiation, the effect of PD98059 $10 \mu \mathrm{M}$ on the ELD of iDCs was investigated. We found that both ERK and CREB phosphorylation was significantly inhibited after a 7-day induction by esophageal carcinoma homogenate in the presence of PD98059 ( $\mathrm{n}=3, \mathrm{P}<0.001$ and $\mathrm{P}<0.01$, respectively) (Fig. 6A). Inhibition of ERK phosphorylation by PD98059 was accompanied by a significantly decreased expression of $\mathrm{vWF}$ in induced cells $(\mathrm{n}=3, \mathrm{P}<0.001)$ (Fig. 6A and B). Furthermore, Dil-Ac-LDL uptake was reduced in the cells of iDC-induced group in the presence of PD98059 (Fig. 6C). Thus, inhibition 
A

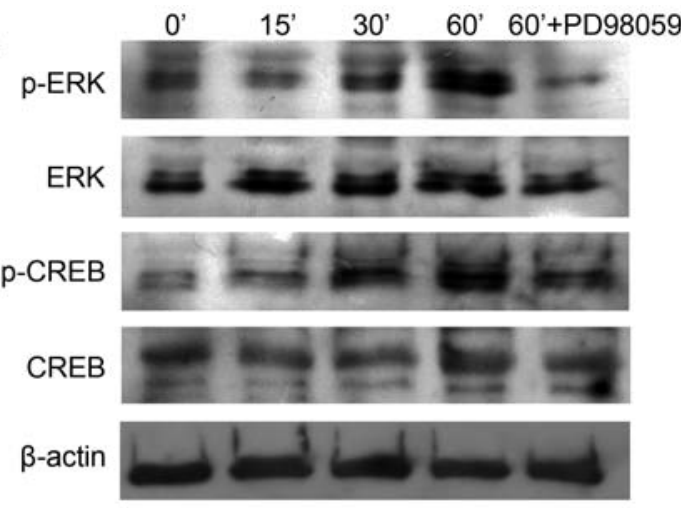

B

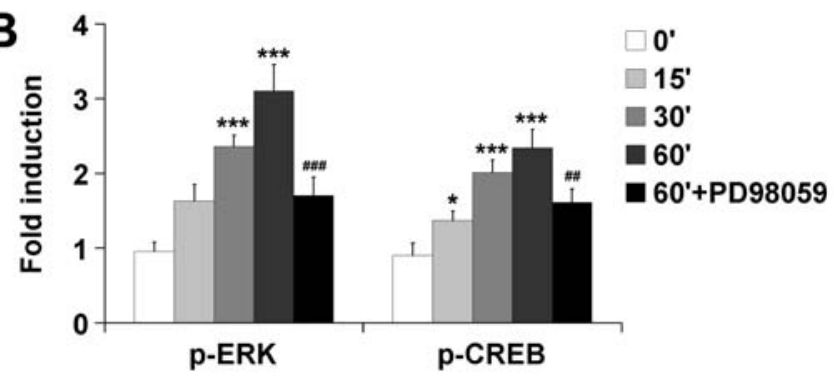

Figure 5. The activation of ERK signaling and CREB induced by esophageal carcinoma tissue homogenate supernatant. ERK and CREB were activated after incubation with esophageal carcinoma homogenate and sustained throughout the period of $60 \mathrm{~min}$ in iDCs, and the activation of ERK and CREB was inhibited by PD98059. Results are means \pm SD $(n=3)$. ${ }^{*} \mathrm{P}<0.05$, ${ }^{* * * *} \mathrm{P}<0.001$ vs. $0^{\prime}$ group. ${ }^{\# \#} \mathrm{P}<0.01,{ }^{\# \# \#} \mathrm{P}<0.001$ vs. 60 ' group. $\mathrm{PD}$ : $\mathrm{PD} 98059$ $50 \mu \mathrm{M}$.

of ERK phosphorylation by PD98059 results in the inhibition of ELD of iDCs.

\section{Discussion}

It is traditionally believed that tumor vascularization is ascribed to the sprouting of ECs from existing vessels. However, recent studies have indicated that the recruitment of endothelial progenitors that differentiate into ECs plays an important role in tumor neovessel formation $(14,15)$. Research has shown that DCs and their precursors might contribute to vasculogenesis by trans-differentiation into ELCs $(6-8,14,16)$. The redirection of differentiation from the DC pathway to ECs might limit the antigen presentation function of DCs, and thereby facilitate tumor growth and immune escape. However, till now the related research has been limited. Therefore, this phenomenon and its mechanisms are worthy of further investigation.

It has been shown that simulating the tumor microenvironment by the presence of cytokines and lactate induced monocytes to transdifferentiate into ELCs in the presence of pro-angiogenic mediators $(8,16)$. CM from murine Lewis lung carcinoma cells was used to demonstrate the $\mathrm{CD} 34^{+}$cell differentiation (7). Our previous research also showed that the CM from EC9706 cell line induced iDCs to differentiate into ELCs (10). However, this kind of CM is far different from the esophageal carcinoma tissue of patients. The potential role of peri-esophageal carcinoma in the ELD of iDCs is also unknown. The growth and development of tumors rely on a continuous cross-talk among cancer cells, together with
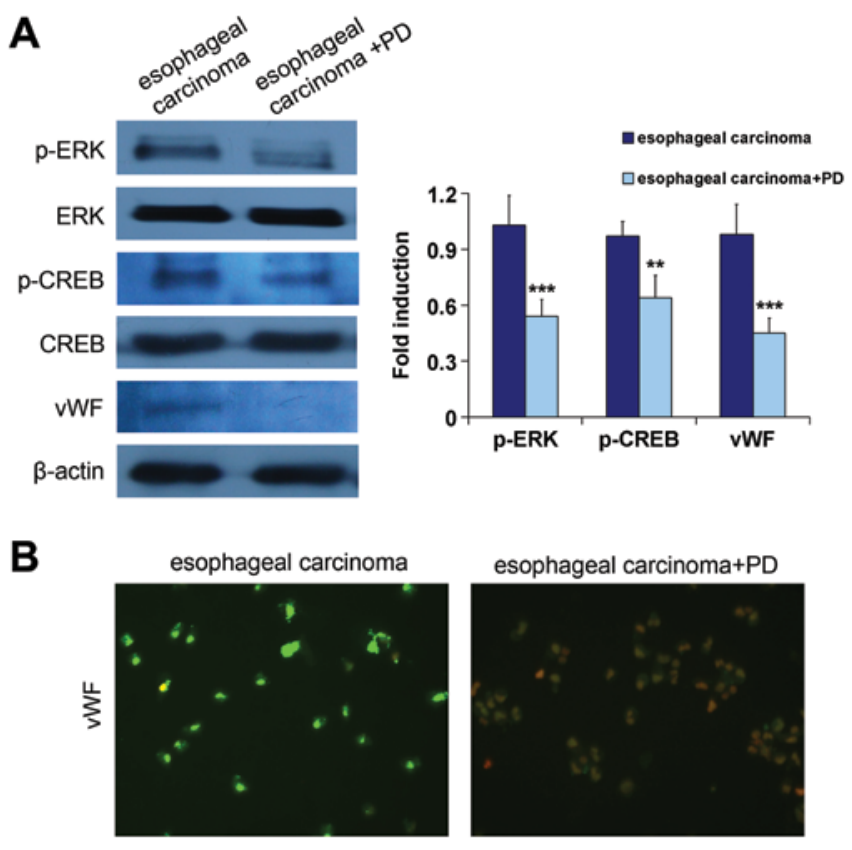

C
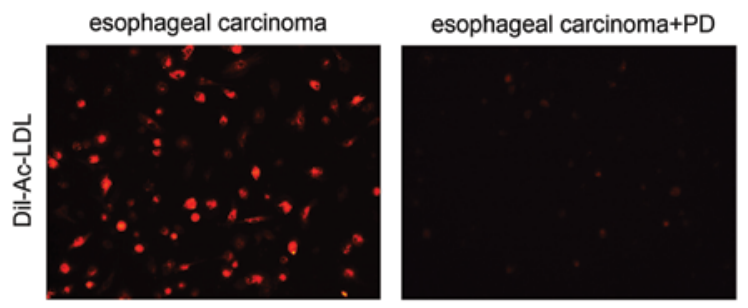

Figure 6. Blocking the activation of ERK signaling and CREB inhibited ELD of iDCs. (A) Adding $10 \mu \mathrm{M}$ PD98059 for 7 days blocked the activation of ERK and CREB, and decreased the expression of vWF in the cells induced by esophageal carcinoma homogenate. Western blot results shown are means $\pm \mathrm{SD}(\mathrm{n}=3) .{ }^{* *} \mathrm{P}<0.01,{ }^{* * *} \mathrm{P}<0.01$ vs. esophageal carcinoma group PD98059 $(10 \mu \mathrm{M})$ decreased the expression of vWF (B) and the Dil-Ac-LDL uptake $(\mathrm{C})$ in the cells induced by esophageal carcinoma homogenate (original magnification, $\mathrm{x} 200)$.

intracellular and extracellular microenvironments, including complicated and mutual interactions among tumor cells, various stromal and other cells $(17,18)$. Therefore, to some extent, tumor tissue homogenate can reflect the tumor microenvironment better than the $\mathrm{CM}$ from cell culture supernatant. Thus, in the present study, we used the homogenate supernatant of human esophageal carcinoma and peri-carcinoma to simulate the tumor microenvironment, to demonstrate iDCs effect on ELD. We found that similar to the CM from EC9706 cell line, esophageal carcinoma homogenate could induce ELD of iDCs. In contrast, peri-carcinoma homogenate did not have this effect. Furthermore, during iDC differentiation into ELCs, the intake of Dil-Ac-LDL, but not India ink, strongly increased in most of the induced cells, distinguishing them from mononuclear cells and macrophages. The activity of APCs obviously decreased, as assessed by checking the capacity to stimulate autologous $\mathrm{T}$ cells in mixed lymphocyte culture and the CTL killing activity. As is known, tumor cells can secrete certain immune factors which can inhibit the development and maturation of DCs $(19,20)$. Some studies have shown that tumor patients have defects in DC maturation, and the reduced function of DCs leads to immune suppression $(21,22)$. The 
redirection of differentiation from DC pathway to ECs might demonstrate one of the mechanisms of the reduced number of tumor-infiltrating DCs.

Many growth factors, cytokines and vasoactive substances can lead to cell differentiation via cellular signal transduction. The MAPK/ERK signaling pathway is involved in cell growth, differentiation, proliferation and apoptosis in multiple physiological processes $(23,24)$. Activated MAPK can phosphorylate specific substrates on serine and/or threonine residues, leading to the activation of various transcription factors to control certain important physiological activities (25). CREB is one of the downstream signal molecules of ERK signaling pathway $(26,27)$. The phosphorylation of CREB mediated by ERK signaling responds to a variety of external signals to regulate cell differentiation and neurite outgrowth $(28,29)$. In the present study, our results demonstrated that ERK phosphorylation was required for ELD of iDCs. PD98059, an inhibitor of MEK, not only inhibited the activation of ERK, but also inhibited the ELD of iDCs, and CREB is involved in this process.

In summary, our results demonstrated that iDCs could differentiate into ELCs under the influence of tissue homogenate of human esophageal carcinoma, while the periesophageal carcinoma homogenate does not have this function. During the course of this differentiation, ERK signaling pathway and CREB were activated to mediate ELD of iDCs. These data suggest that esophageal carcinoma tissue can drive iDCs to differentiate into ELCs, instead of differentiation into mature DCs, thereby losing its ability of antigen presentation.

\section{Acknowledgements}

This study was supported by the Natural Science Foundation of China (no. 81101731).

\section{References}

1. Xue G, Cheng Y, Ran F, et al: SLC gene-modified dendritic cells mediate $\mathrm{T}$ cell-dependent anti-gastric cancer immune responses in vitro. Oncol Rep 29: 595-604, 2013.

2. Wu H, Han Y, Qin Y, et al: Whole-cell vaccine coated with recombinant calreticulin enhances activation of dendritic cells and induces tumour-specific immune responses. Oncol Rep 29: 529-534, 2013.

3. Giardino Torchia ML, Ciaglia E, Masci AM, et al: Dendritic cells/natural killer cross-talk: a novel target for human immunodeficiency virus type-1 protease inhibitors. PLoS One 5: e11052, 2010.

4. Demoulin S, Herfs M, Delvenne P and Hubert P: Tumor microenvironment converts plasmacytoid dendritic cells into immunosuppressive/tolerogenic cells: insight into the molecular mechanisms. J Leukoc Biol 93: 343-352, 2012.

5. Benencia F, Sprague L, McGinty J, Pate M and Muccioli M: Dendritic cells the tumor microenvironment and the challenges for an effective antitumor vaccination. J Biomed Biotechnol 2012: 425476, 2012.

6. Conejo-Garcia JR, Benencia F, Courreges MC, et al: Tumorinfiltrating dendritic cell precursors recruited by a $\beta$-defensin contribute to vasculogenesis under the influence of Vegf-A. Nat Med 10: 950-958, 2004.

7. Young MR and Cigal M: Tumor skewing of CD $34^{+}$cell differentiation from a dendritic cell pathway into endothelial cells. Cancer Immunol Immunother 55: 558-568, 2006.

8. Gottfried E, Kreutz M, Haffner S, et al: Differentiation of human tumour-associated dendritic cells into endothelial-like cells: an alternative pathway of tumour angiogenesis. Scand J Immunol 65: 329-335, 2007.
9. Sozzani S, Rusnati M, Riboldi E, Mitola S and Presta M: Dendritic cell-endothelial cell cross-talk in angiogenesis. Trends Immunol 28: 385-392, 2007.

10. Lu J, Zhao J, Liu K, et al: MAPK/ERK1/2 signaling mediates endothelial-like differentiation of immature DCs in the microenvironment of esophageal squamous cell carcinoma. Cell Mol Life Sci 67: 2091-2106, 2010.

11. Muftuoglu TM, Koksal N and Ozkutlu D: Evaluation of phagocytic function of macrophages in rats after partial splenectomy. $\mathrm{J}$ Am Coll Surg 191: 668-671, 2000.

12. Aranguren XL, Luttun A, Clavel $\mathrm{C}$, et al: In vitro and in vivo arterial differentiation of human multipotent adult progenitor cells. Blood 109: 2634-2642, 2007.

13. Li Z, Wu JC, Sheikh AY, et al: Differentiation, survival, and function of embryonic stem cell derived endothelial cells for ischemic heart disease. Circulation 116: I46-I54, 2007.

14. Conejo-Garcia JR, Buckanovich RJ, Benencia F, et al: Vascular leukocytes contribute to tumor vascularization. Blood 105: 679-681, 2005.

15. Rehman J, Li J, Orschell CM and March KL: Peripheral blood 'endothelial progenitor cells' are derived from monocyte/macrophages and secrete angiogenic growth factors. Circulation 107: 1164-1169, 2003.

16. Fernandez Pujol B, Lucibello FC, Zuzarte M, Lutjens P, Muller R and Havemann K: Dendritic cells derived from peripheral monocytes express endothelial markers and in the presence of angiogenic growth factors differentiate into endothelial-like cells. Eur J Cell Biol 80: 99-110, 2001.

17. Said N and Theodorescu D: RhoGDI2 suppresses bladder cancer metastasis via reduction of inflammation in the tumor microenvironment. Oncoimmunology 1: 1175-1177, 2012.

18. Fessler E, Dijkgraaf FE, De Sousa EMF and Medema JP: Cancer stem cell dynamics in tumor progression and metastasis: is the microenvironment to blame? Cancer Lett 12: 00603-00609, 2012.

19. Kusmartsev S and Gabrilovich DI: Effect of tumor-derived cytokines and growth factors on differentiation and immune suppressive features of myeloid cells in cancer. Cancer Metastasis Rev 25: 323-331, 2006.

20. Muthana M, Fairburn B, Mirza S, Slack LK, Hopkinson K and Pockley AG: Identification of a rat bone marrow-derived dendritic cell population which secretes both IL-10 and IL-12: evidence against a reciprocal relationship between IL-10 and IL-12 secretion. Immunobiology 211: 391-402, 2006.

21. Michielsen AJ, O'Sullivan JN and Ryan EJ: Tumor conditioned media from colorectal cancer patients inhibits dendritic cell maturation. Oncoimmunology 1: 751-753, 2012.

22. Hurwitz AA and Watkins SK: Immune suppression in the tumor microenvironment: a role for dendritic cell-mediated tolerization of $\mathrm{T}$ cells. Cancer Immunol Immunother 61: 289-293, 2012.

23. Tamminen JA, Myllarniemi M, Hyytiainen M, Keski-Oja J and Koli K: Asbestos exposure induces alveolar epithelial cell plasticity through MAPK/Erk signaling. J Cell Biochem 113: 2234-2247, 2012.

24. Shi Y, Xia YY, Wang L, Liu R, Khoo KS and Feng ZW: Neural cell adhesion molecule modulates mesenchymal stromal cell migration via activation of MAPK/ERK signaling. Exp Cell Res 318: 2257-2267, 2012.

25. Wu W, Sun Z, Wu J, et al: Trihydrophobin 1 phosphorylation by c-Src regulates MAPK/ERK signaling and cell migration. PLoS One 7: e29920, 2012.

26. Gomez M, Manzano A, Figueras A, et al: Sertoli-secreted FGF-2 induces PFKFB4 isozyme expression in mouse spermatogenic cells by activation of the MEK/ERK/CREB pathway. Am J Physiol Endocrinol Metab 303: E695-E707, 2012.

27. Guo Y and Feng P: OX2R activation induces PKC-mediated ERK and CREB phosphorylation. Exp Cell Res 318: 2004-2013, 2012.

28. Kido S, Kuriwaka-Kido R, Imamura T, Ito Y, Inoue D and Matsumoto T: Mechanical stress induces Interleukin-11 expression to stimulate osteoblast differentiation. Bone 45: 1125-1132, 2009.

29. Choi HJ, Park YG and Kim CH: Lactosylceramide alpha2,3sialyltransferase is induced via a PKC/ERK/CREB-dependent pathway in K562 human leukemia cells. Mol Cells 23: 138-144, 2007. 